\title{
3-D MIMO Radar Imaging of Ship Target with Rotational Motions
}

\author{
Wei WANG, Ziying HU, Ping HUANG \\ Automation College, Harbin Engineering University, Nantong Street 145, 150001 Harbin, China \\ wangwei407@hrbeu.edu.cn, huziying@hrbeu.edu.cn, hppmonkeyking@163.com
}

Submitted April 7, 2019 / Accepted September 16, 2019

\begin{abstract}
The problem of image defocusing and distortion occurs in synthetic aperture radar (SAR) imaging of ship target with rotations. Although many literatures have analyzed this problem, it cannot efficiently be solved due to the coherent accumulation time for SAR target imaging, which results from their working principle and mechanism. In this paper, three-dimensional MIMO radar imaging of ship target with rotations is proposed. The real-time advantage of MIMO radar imaging is utilized to achieve high-precision imaging of ship target with rotations. Firstly, the ship rotations characteristics and imaging distortion effects are analyzed in detail. Then, the three-dimensional MIMO radar echo model is built to correct the scattering positions in time, geometrical analysis is carried out to analyze and optimize the target scattering coefficient variation process. Finally, a sparse imaging algorithm based on maximum a posterior (MAP) method is proposed to obtain accurate imaging result. Theoretical analysis and simulation results show that the three-dimensional imaging of ship target based on MIMO radar has better imaging accuracy and real-time performance compared with SAR, and can effectively solve the problem of image defocusing and distortion.
\end{abstract}

\section{Keywords}

Ship rotations, MIMO radar imaging, geometrical analysis, real-time performance

\section{Introduction}

Image defocusing or distortion problem results from the complex motions of ship has been a great challenge for the research in radar imaging, particularly the SAR imaging [1-3]. Moreover, the error caused by imaging distortion effects will become larger and larger with time accumulate. Therefore, the accuracy and real-time performance of SAR imaging cannot be guaranteed [4-5].

This problem has received great research attentions in recent years. In [6], SAR images are obtained based on measured data and known parameters, the influence of pitch motion on SAR imaging is analyzed. However, the lackness of measured data greatly limits its development, so it is necessary to describe the ship motions in detail for further studies by building motion model. A virtual model of ship is built in [7] and SAR image is obtained by the simulation device [8]. But there was no effective method to optimize imaging performance. For this purpose, [9] and [10] built 3-D geometry scenario models, and optimized the imaging result by combining SAR and ISAR system. A method of recording Doppler history in each resolution unit is proposed in [11]. The dynamic models of ship roll, pitch and yaw rotations are established in [12]. Based on these, paper [13] analyzes the imaging result on range bin and azimuth bin of SAR imaging, and proposes a compensation method to improve imaging performance by adding phase profile to azimuth sampling. Although the above method can achieve better imaging effect, the original imaging defocusing problem can not be completely solved. On one hand, the rotation effects can not be accurately compensated because the synchronization between the measured data and the ship attitude changes cannot be guaranteed, on the other hand, the migration through resolution cell (MTRC) problem will further aggravately deteriorate imaging performance.

Multi-input multi-output (MIMO) radar is a radar system with a special working mechanism. It transmits orthogonal signals at the transmitter and separates channels through matched filtering at the receiver [14-17]. It can obtain more virtual aperture and array freedom. Moreover, single snapshot real-time imaging can greatly improve the performance of radar imaging [18-22]. For rotational target, literatures [23-25] have carried out relevant studies on the estimation of rotational parameters and target positions, which can improve the accuracy and reliability. However, these literatures mostly focus on one or two dimensional rotations under ideal conditions, and optimize the signal geometric model or combining the processing method of SAR or ISAR system with MIMO radar imaging, which is hard to fundamentally realize real-time accurate imaging for target with 3-D rotations.

This paper presents a high-precision three-dimensional (3-D) imaging method for ship target based on MIMO radar. Firstly, the 3-D rotations characteristics of ship target are 
analyzed in detail. Secondly, according to the real-time changes of scattering positions, MIMO radar echo model based on ship rotations is established, and the scintillation problem caused by the change of scattering coefficient under complex motion conditions is analyzed and optimized geometrically. Then, the maximum a posteriori (MAP) algorithm is used to accurately recover the sparse signal, then imaging results can be obtained. Finally, the simulation results show that three-dimensional imaging based on MIMO radar can visually display the moving state of ship targets, and it has higher accuracy and efficiency than SAR imaging.

This paper is organized as follows. In Sec. 2, the rotational motions of ship are analyzed and a motion model is established based on [12]. In Sec. 3, a three-dimensional echo model of MIMO radar is build based on vertical array structure, and the change process of target scattering coefficient is analyzed and optimized, then the MAP method is used to achieve accurate three-dimensional sparse imaging. In Sec. 4, simulation results and discussion are given to illustrate the performance advantages of MIMO radar imaging. Finally, Section 5 gives some conclusions.

\section{Analysis of Ship Rotations}

In the course of navigation, besides moving along the cruise direction, ships will also be affected by strong external forces (such as water, waves and sea breezes), which will generate additional motions, such as pitch, roll and yaw. These rotations will cause continuous changes in the scattering position and radar cross section (RCS) of the target, and make the radar image produce virtual shadow or defocus. Figure 1 shows the three forms, respectively.

Considering that there is no cruise motion in the marine environment but three-axis harmonic motions, namely pitch, roll and yaw. According to [13], these motion forms are typical sinusoidal, which can be written as following:

$$
\begin{aligned}
& \theta_{\mathrm{p}}(t)=A_{\mathrm{p}} \sin \left(\frac{2 \pi}{T_{\mathrm{p}}} t+\gamma_{\mathrm{p}}\right), \\
& \theta_{\mathrm{r}}(t)=A_{\mathrm{r}} \sin \left(\frac{2 \pi}{T_{\mathrm{r}}} t+\gamma_{\mathrm{r}}\right), \\
& \theta_{\mathrm{y}}(t)=A_{\mathrm{y}} \sin \left(\frac{2 \pi}{T_{\mathrm{y}}} t+\gamma_{\mathrm{y}}\right)
\end{aligned}
$$

where $\theta_{\mathrm{p}}(t), \theta_{\mathrm{r}}(t)$ and $\theta_{\mathrm{y}}(t)$ represent the time-varying angles along the axis of the ship caused by pitch, roll and yaw rotations, $A_{\mathrm{p}}(t), A_{\mathrm{r}}(t), A_{\mathrm{y}}(t), T_{\mathrm{p}}(t), T_{\mathrm{r}}(t), T_{\mathrm{y}}(t)$ and $\gamma_{\mathrm{p}}(t), \gamma_{\mathrm{r}}(t)$, $\gamma_{\mathrm{y}}(t)$ represent the maximum amplitudes, periods and the initial phases of the corresponding rotation respectively. The above formulas describe the specific forms of the rotational motions and corresponding rotation matrixes under pitch, roll and yaw rotations can be obtained as

$$
\begin{aligned}
\operatorname{pitch}\left(\theta_{\mathrm{p}}(t)\right) & =\left[\begin{array}{ccc}
1 & 0 & 0 \\
0 & \cos \theta_{\mathrm{p}}(t) & -\sin \theta_{\mathrm{p}}(t) \\
0 & \sin \theta_{\mathrm{p}}(t) & \cos \theta_{\mathrm{p}}(t)
\end{array}\right], \\
\operatorname{roll}\left(\theta_{\mathrm{r}}(t)\right) & =\left[\begin{array}{ccc}
\cos \theta_{\mathrm{r}}(t) & 0 & \sin \theta_{\mathrm{r}}(t) \\
0 & 1 & 0 \\
-\sin \theta_{\mathrm{r}}(t) & 0 & \cos \theta_{\mathrm{r}}(t)
\end{array}\right], \\
\operatorname{yaw}\left(\theta_{\mathrm{y}}(t)\right) & =\left[\begin{array}{ccc}
\cos \theta_{\mathrm{y}}(t) & -\sin \theta_{\mathrm{y}}(t) & 0 \\
\sin \theta_{\mathrm{y}}(t) & \cos \theta_{\mathrm{y}}(t) & 0 \\
0 & 0 & 1
\end{array}\right] .
\end{aligned}
$$

Thus synthetic rotation matrix can be expressed as

$$
\boldsymbol{\Omega}=\operatorname{pitch}\left(\theta_{\mathrm{p}}(t)\right) \cdot \operatorname{roll}\left(\theta_{\mathrm{r}}(t)\right) \cdot \operatorname{yaw}\left(\theta_{\mathrm{y}}(t)\right) .
$$

Firstly, the rotations cause continuous changes of scattering position, which results in position errors cannot be ignored for MIMO radar target accurate imaging and localization [23-25]. In order to accurately represent the real-time position of each scattering point, a reference coordinate system located on the radar platform (OXYZ coordinate system in Fig. 2) needs to be selected beforehand. Here, taking $\left[x_{0}, y_{0}, z_{0}\right]^{\mathrm{T}}$ as the initial coordinate of any point on board, then time-varying coordinate system can be obtained from the formula:

$$
\left[\begin{array}{l}
x(t) \\
y(t) \\
z(t)
\end{array}\right]=\boldsymbol{\Omega} \cdot\left[\begin{array}{l}
x_{0} \\
y_{0} \\
z_{0}
\end{array}\right] .
$$

At the same time, the rotations cause continuous changes of the scattering angle of the target, and then change the radar cross section of each scattering point, which affects the scattering coefficient detected by the radar, and ultimately leads to the glint problem of the ship target imaging. So it is essential to solve this problem.

It should be noted that in addition to pitch, roll and yaw rotations, there are other three translational motions, namely heave, surge and sway, which are not considered in the above description because they only affect the effective cruise speed of the ship, but have a relatively small impact on the radial speed and image offset of the ship [13].

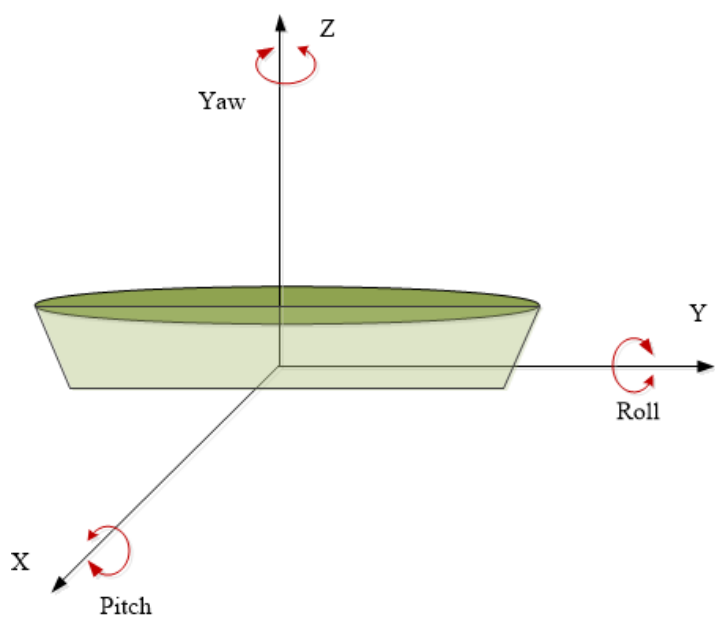

Fig. 1. Pitch, roll and yaw rotations of ship target. 


\section{MIMO Radar 3-D Imaging of Ship Target with Rotational Motions}

\subsection{MIMO Radar 3-D Echo Model}

For 3-D imaging of ship target, an appropriate array layout is required for MIMO radar. The transmitting array used in the paper is an uniform linear array composed of $M$ transmitters. The $i$-th antenna is represented by $T_{i}$, and the corresponding transmitted signal $s_{i}(t)=p_{i}(t) \exp \left(\mathrm{j} 2 \pi f_{\mathrm{c}} t+\varphi_{i}\right)$ is Hadamard orthogonal-phase encoded signal, where $p_{i}(t)$ is the complex envelope of signal, $f_{\mathrm{c}}$ is the carrier frequency of transmitted signal and $\varphi_{i}$ is the phase of signal. The orthogonality of each transmitting signal is guaranteed by adjusting the phase $\varphi_{i}$. As for the receiving array, an uniform planar array is adopt in the paper which is divided into $N_{\mathrm{R}}$ rows and $N_{\mathrm{C}}$ columns, and the antenna located in the $q$-th row, $l$-th column is represented by $R_{q l}$. The distribution of the transmitting array is perpendicular to the plane of the receiving array, as shown in Fig. 2, the common reference antenna of two arrays is located at the origin of coordinates, and the linear direction of the transmitting array is taken as the $\mathrm{X}$-axis direction, and the linear directions of the receiving array are taken as the axial directions of $\mathrm{Y}$ and $\mathrm{Z}$ respectively, then the reference coordinate system is established as Fig. 2

After the transmitting signal of $T_{i}$ is reflected by all of $\mathrm{K}$ scattering points, the echo signal at $R_{q l}$ can be expressed as

$$
d_{i, q, l}(t)=\sum_{k=1}^{K} \sigma_{k} p_{i}\left(t-\tau_{k, i, q, l}\right) \cdot \exp \left[\mathrm{j} 2 \pi f_{\mathrm{c}}\left(t-\tau_{k, i, q, l}\right)\right]
$$

where $\sigma_{k}$ is the scattering coefficient of the $k$-th scattering point, the delay time is $\tau_{k, i, q, l}=\left(T_{i} k+R_{q l} k\right) / \mathrm{c}$ and $\mathrm{c}$ is the propagation speed of the electromagnetic signal, $T_{i} k$ represents the distance from the $i$-th transmitter to the $k$-th target and $R_{q l} k$ represents the distance from the $k$-th target to the $q l$-th receiver. Considering that the main lobe of echo signals from different transceiver channels are in a same range bin, which means there is no effect of range-cross, thus consider that there is only the main lobe of the signal in range bin. Therefore, after removing carrier processing, the $i$-th transmitting signal at $q l$-th receiver can be written as

$$
d_{i, q, l}=\sum_{k=1}^{K} \sigma_{k} \cdot \exp \left[-\mathbf{j} 2 \pi\left(\left|\overrightarrow{\mathbf{T}_{\mathbf{i}} \mathbf{k}}\right|+\left|\overrightarrow{\mathbf{R}_{\mathbf{q} \mathbf{l}}}\right|\right) / \lambda\right]
$$

where $\lambda$ is the signal wavelength and $\left|\overrightarrow{\mathbf{T}_{\mathbf{i}} \mathbf{k}}\right|=T_{i} k,\left|\overrightarrow{\mathbf{R}_{\mathbf{q} \mathbf{l}} \mathbf{k}}\right|=$ $R_{q l} k$. Then, suppose $\mathrm{P}$ is the reference center in the imaging region, and its coordinate in the OXYZ coordinate system is $\left(P_{\mathrm{x}}, P_{\mathrm{y}}, P_{\mathrm{z}}\right)$. Let $\left|\overrightarrow{\mathbf{T}_{\mathbf{i}} \mathbf{P}}\right|$ and $\left|\overrightarrow{\mathbf{R}_{\mathbf{q}} \mathbf{P}}\right|$ respectively represents the distance from $\mathrm{P}$ to the $i$-th transmiter and $q l$-th receiver, then the echo signal reflected from $P$ can be used as a reference signal to compensate the target echo signal:

$$
\begin{aligned}
& D_{i, q, l}=d_{i, q, l} \times d_{\mathrm{P}, i, q, l}^{*} \\
& =\sum_{k=1}^{K} \sigma_{k} \cdot \exp \left[-\mathbf{j} 2 \pi\left(\left|\overrightarrow{\mathbf{T}_{\mathbf{i}} \mathbf{k}}\right|+\left|\overrightarrow{\mathbf{R}_{\mathbf{q}} \mathbf{k}}\right|-\left|\overrightarrow{\mathbf{T}_{\mathbf{i}} \mathbf{P}}\right|-\left|\overrightarrow{\mathbf{R}_{\mathbf{q}} \mathbf{P}}\right|\right) / \lambda\right]
\end{aligned}
$$

According to the geometrical model shown in Fig. 2, let $\Delta R$ represents the range profile in (7), and it can be further processed as

$$
\begin{aligned}
& \Delta R=\left|\overrightarrow{\mathbf{T}_{\mathbf{i}} \mathbf{k}}\right|+\left|\overrightarrow{\mathbf{R}_{\mathbf{q} \mathbf{l}} \mathbf{k}}\right|-\left(\left|\overrightarrow{\mathbf{T}_{\mathbf{i}} \mathbf{P}}\right|+\left|\overrightarrow{\mathbf{R}_{\mathbf{q} \mathbf{l}} \mathbf{P}}\right|\right) \\
& =\left(\left|\overrightarrow{\mathbf{T}_{\mathbf{i}} \mathbf{k}}\right|-\left|\overrightarrow{\mathbf{T}_{\mathbf{i}} \mathbf{P}}\right|\right)+\left(\left|\overrightarrow{\mathbf{R}_{\mathbf{q} \mathbf{l}} \mathbf{k}}\right|-\left|\overrightarrow{\mathbf{R}_{\mathbf{q} \mathbf{l}} \mathbf{P}}\right| .\right.
\end{aligned}
$$

In the formula, as for $\left|\overrightarrow{\mathbf{T}_{\mathbf{i}} \mathbf{k}}\right|-\left|\overrightarrow{\mathbf{T}_{\mathbf{i}} \mathbf{P}}\right|=\left|\overrightarrow{\mathbf{T}_{\mathbf{i}} \mathbf{P}}+\overrightarrow{\mathbf{P k}}\right|-\left|\overrightarrow{\mathbf{T}_{\mathbf{i}} \mathbf{P}}\right|$ and $\left|\overrightarrow{\mathbf{R}_{\mathbf{q} \mathbf{l}} \mathbf{k}}\right|-\left|\overrightarrow{\mathbf{R}_{\mathbf{q} \mathbf{P}} \mathbf{P}}\right|=\left|\overrightarrow{\mathbf{R}_{\mathbf{q l}} \mathbf{P}}+\overrightarrow{\mathbf{P k}}\right|-\left|\overrightarrow{\mathbf{R}_{\mathbf{q} \mathbf{l}} \mathbf{P}}\right|$, due to $|\overrightarrow{\mathbf{P k}}|<<\left|\overrightarrow{\mathbf{T}_{\mathbf{i}} \mathbf{P}}\right|$ and $|\overrightarrow{\mathbf{P k}}|<<\left|\overrightarrow{\mathbf{R}_{\mathbf{q l}} \mathbf{P}}\right|$, we can get the following approximation using first-order Taylor expansion:

$$
\mid \begin{aligned}
& \overrightarrow{\mathbf{T}_{\mathbf{i}} \mathbf{P}}+\overrightarrow{\mathbf{P k}}|\approx| \overrightarrow{\mathbf{T}_{\mathbf{i}} \mathbf{P}} \mid+\left(\overrightarrow{\mathbf{T}_{\mathbf{i}} \mathbf{P}}\right)^{\prime} \cdot \overrightarrow{\mathbf{P k}}, \\
& \overrightarrow{\mathbf{R}_{\mathbf{q} \mathbf{l}} \mathbf{P}}+\overrightarrow{\mathbf{P k}}|\approx| \overrightarrow{\mathbf{R}_{\mathbf{q} \mathbf{l}} \mathbf{P}} \mid+\left(\overrightarrow{\mathbf{R}_{\mathbf{q}} \mathbf{P}}\right)^{\prime} \cdot \overrightarrow{\mathbf{P k}}
\end{aligned}
$$

where $\left(\overrightarrow{\mathbf{T}_{\mathbf{i}} \mathbf{P}}\right)^{\prime}$ and $\left(\overrightarrow{\mathbf{R}_{\mathbf{q l}} \mathbf{P}}\right)^{\prime}$ respectively represent the unit direction vector from the transmitter and receiver to the reference center $P$, then equation (8) can be expressed as

$$
\Delta R \approx\left[\left(\overrightarrow{\mathbf{T}_{\mathbf{i}} \mathbf{P}}\right)^{\prime}+\left(\overrightarrow{\mathbf{R}_{\mathbf{q l}} \mathbf{P}}\right)^{\prime}\right] \cdot \overrightarrow{\mathbf{P k}}
$$

Then, we set the coordinates of point $\mathrm{k}$ is $\left(P_{\mathrm{x}}+x_{\mathrm{k}}, P_{\mathrm{y}}+\right.$ $\left.y_{\mathrm{k}}, P_{\mathrm{z}}+z_{\mathrm{k}}\right)$ in the OXZY coordinate system, $d_{\mathrm{x}}$ is the internal spacing of the transmitting array, $d_{\mathrm{y}}$ and $d_{\mathrm{z}}$ respectively denote the row and column spacing inside the receiving array, then the coordinate of $T_{i}$ transmitter is $\left((i-1) d_{\mathrm{x}}, 0,0\right)$, and the coordinate of $R_{q l}$ receiver is $\left(0,(q-1) d_{\mathrm{y}},(l-1) d_{\mathrm{z}}\right)$. Therefore we can get that $\left(\overrightarrow{\mathbf{T}_{\mathrm{i}} \mathbf{P}}\right)^{\prime} \approx\left(P_{\mathrm{x}}-(i-1) d_{\mathrm{x}}, P_{\mathrm{y}}, P_{\mathrm{z}}\right) / R_{0}$ and $\left(\overrightarrow{\mathbf{R}_{\mathbf{q} \mathbf{l}} \mathbf{P}}\right)^{\prime} \approx\left(P_{\mathrm{x}}, P_{\mathrm{y}}-(q-1) d_{\mathrm{y}}, P_{\mathrm{z}}-(l-1) d_{\mathrm{z}}\right) / R_{0}$, where $R_{0}$ is the reference distance from the reference center $\mathrm{P}$ to the coordinate center O. According to (4), (7), (10), the real-time echo model can be written as

$$
\begin{aligned}
& D_{i, q, l}=\sum_{k}^{K} \sigma_{k} \exp \left(\mathrm{j} 2 \pi \frac{2 P_{\mathrm{x}}-(i-1) d_{\mathrm{x}}}{\lambda \cdot R_{0}} x_{k}(t)\right) \\
& \cdot \exp \left(\mathrm{j} 2 \pi \frac{2 P_{\mathrm{y}}-(q-1) d_{\mathrm{y}}}{\lambda \cdot R_{0}} y_{k}(t)\right) \cdot \exp \left(\mathrm{j} 2 \pi \frac{2 P_{\mathrm{z}}-(l-1) d_{\mathrm{z}}}{\lambda \cdot R_{0}} z_{k}(t)\right)
\end{aligned}
$$

where $t$ is the time the ship moves.

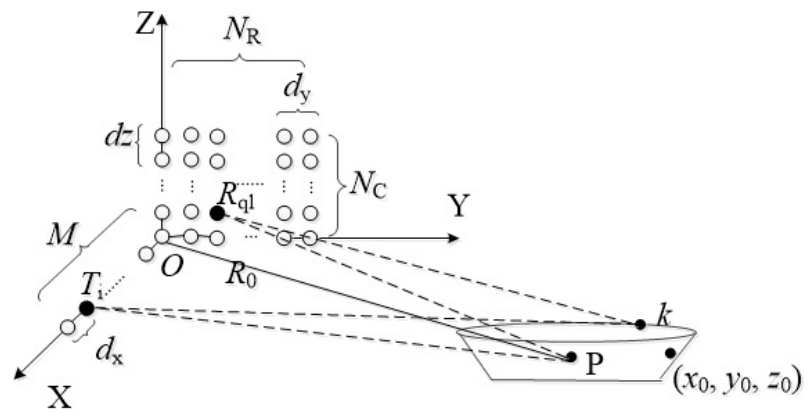

Fig. 2. MIMO radar array structure and imaging model. 


\subsection{Geometrical Analysis for the Scintillation Problem}

Under ocean conditions, the scattering coefficient changes due to the continuous changes of radar cross section (RCS), so the rotations of ship will cause the scattering scintillation problem. Therefore, based on the proportional relationship between scattering coefficient and radar cross section, the change of scattering coefficient is analyzed and optimized geometrically in this section.

We choose the simplest triangle to analyze this problem, as is shown in Fig. 3, triangle A is the RCS of $k$-th target where point $k$ is the center and a, b, c are the vertexes, respectively. $\mathbf{r}_{\mathbf{1}}$ is the normal vector of the plane which represents the direction of radar view, considered as a constant vector due to the long distance between target and radar system. But in actual, after complex motions of the ship, A moves to triangle $\mathrm{B}$ with a new center $k_{0}$. Considering that the RCS of two triangles are $S_{1}$ and $S_{2}$, in the line of radar view $\mathbf{r}_{1}$, area of A is its own RCS, but the RCS of B in this direction is its area projection along the vector $\mathbf{r}_{1}$. In Fig. $3, \mathbf{r}_{2}$ is the normal vector of triangle $\mathrm{B}$, so the angle between two planes can be represented by $\mathbf{r}_{\mathbf{1}}$ and $\mathbf{r}_{2}$, which can be expressed as

$$
\beta=\arccos \frac{\mathbf{r}_{1} \cdot \mathbf{r}_{2}}{\left|\mathbf{r}_{1}\right| \cdot\left|\mathbf{r}_{2}\right|} .
$$

So we can get the projection area of B

$$
S_{2}=S_{1} \cdot \cos \beta
$$

For $\mathbf{r}_{1}$ and $\mathbf{r}_{2}$, we can get

$$
\left\{\begin{array} { l } 
{ \mathbf { r } _ { 1 } \cdot \mathbf { a b } = 0 } \\
{ \mathbf { r } _ { 1 } \cdot \mathbf { b c } = 0 } \\
{ \mathbf { r } _ { 1 } \cdot \mathbf { a c } = 0 }
\end{array} \quad \text { and } \left\{\begin{array}{l}
\mathbf{r}_{2} \cdot \mathbf{a}^{\prime} \mathbf{b}^{\prime}=0 \\
\mathbf{r}_{2} \cdot \mathbf{b}^{\prime} \mathbf{c}^{\prime}=0 \\
\mathbf{r}_{2} \cdot \mathbf{a}^{\prime} \mathbf{c}^{\prime}=0
\end{array} \quad .\right.\right.
$$

Due to $a^{\prime}=\boldsymbol{\Omega} \cdot a, b^{\prime}=\boldsymbol{\Omega} \cdot b, c^{\prime}=\boldsymbol{\Omega} \cdot c$, according to above formulas, $\mathbf{r}_{\mathbf{1}}$ and $\mathbf{r}_{\mathbf{2}}$ satisfy $\mathbf{r}_{\mathbf{2}}=\boldsymbol{\Omega} \cdot \mathbf{r}_{\mathbf{1}}$, then equation (12) can be written as

$$
\cos \beta=\frac{\mathbf{r}_{\mathbf{1}} \cdot\left(\boldsymbol{\Omega} \cdot \mathbf{r}_{1}\right)}{\left|\mathbf{r}_{\mathbf{1}}\right| \cdot\left|\boldsymbol{\Omega} \cdot \mathbf{r}_{1}\right|} .
$$

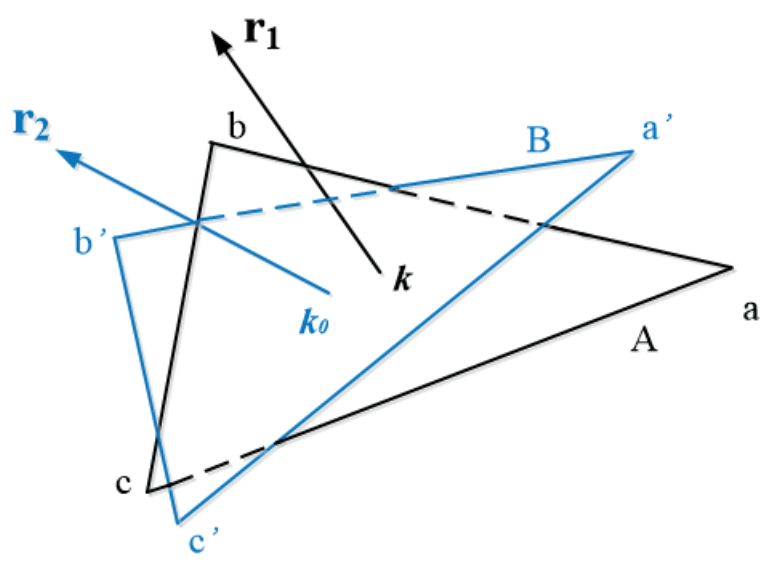

Fig. 3. Change of RCS due to complex rotations.
Combining equation (15) and the geometrical model in Fig. 1, the stable scattering coefficient of $k$-th target is $\sigma_{k}$ along direction of $\mathbf{r}_{1}$, then let $\beta_{k}$ represent the corresponding angle, it is convenient to obtain the actual scattering coefficient by

$$
\alpha_{k}=\sigma_{k} \cdot \cos \beta_{k}
$$

So the model in (11) is transformed into

$$
\begin{aligned}
& D_{i, q, l}=\sum_{k}^{K} \alpha_{k} \exp \left(\mathrm{j} 2 \pi \frac{2 P_{\mathrm{x}}-(i-1) d_{\mathrm{x}}}{\lambda \cdot R_{0}} x_{k}(t)\right) \\
& \cdot \exp \left(\mathrm{j} 2 \pi \frac{2 P_{\mathrm{y}}-(q-1) d_{\mathrm{y}}}{\lambda \cdot R_{0}} y_{k}(t)\right) \cdot \exp \left(\mathrm{j} 2 \pi \frac{2 P_{\mathrm{z}}-(l-1) d_{\mathrm{z}}}{\lambda \cdot R_{0}} z_{k}(t)\right) .
\end{aligned}
$$

\subsection{MIMO Radar 3-D Imaging Based on MAP Method}

Taking the reference point $\mathrm{P}$ on the ship as the central point to construct the spatial area as shown in Fig. 4, the target area is composed of $U=U_{\mathrm{X}} U_{\mathrm{Y}} U_{\mathrm{Z}}$ grids, which is divided along the axis directions of $\mathrm{X}, \mathrm{Y}$ and $\mathrm{Z}$, and the strong scattering points are set on the surface contour of the ship then the echo model in (17) can be written as

$$
\begin{aligned}
& D_{i, q, l}=\sum_{u}^{U} \alpha_{k} \exp \left(\mathrm{j} 2 \pi \frac{2 P_{\mathrm{x}}-(i-1) d_{\mathrm{x}}}{\lambda \cdot R_{0}} x_{u}(t)\right) \\
& \cdot \exp \left(\mathrm{j} 2 \pi \frac{2 P_{\mathrm{y}}-(q-1) d_{\mathrm{y}}}{\lambda \cdot R_{0}} y_{u}(t)\right) \cdot \exp \left(\mathrm{j} 2 \pi \frac{2 P_{\mathrm{z}}-(l-1) d_{\mathrm{z}}}{\lambda \cdot R_{0}} z_{u}(t)\right) .
\end{aligned}
$$

Based on the mutual decoupling characteristics between each dimension parameters in (18), we search the direction range separately from each dimension. For X dimension, we can get the echo model as

$$
\mathbf{D}_{\mathrm{x}}=\mathbf{A}_{\mathrm{x}} \boldsymbol{\alpha}_{\mathrm{x}}+\mathbf{e}_{\mathrm{x}}
$$

where $\mathbf{D}_{\mathrm{x}}=\left[d_{\mathrm{x}, 1}^{\mathrm{T}}, \cdots, d_{\mathrm{x}, \mathrm{M}}^{\mathrm{T}}\right]^{\mathrm{T}} \in \mathrm{C}^{\mathrm{M} \times 1}$ and $\boldsymbol{\alpha}_{\mathrm{x}}=$ $\left[\alpha_{\mathrm{x}, 1}, \cdots, \alpha_{\mathrm{x}, \mathrm{U}_{\mathrm{x}}}\right]^{\mathrm{T}} \in \mathrm{C}^{\mathrm{U}_{\mathrm{x}} \times 1}$ respectively represents the echo and scattering vector in $\mathrm{X}$ dimension, $\mathbf{A}_{\mathrm{x}} \in \mathrm{C}^{\mathrm{M} \times \mathrm{U}_{\mathrm{x}}}$ is measurement matrix in $\mathrm{X}$ dimension, $\mathbf{e}_{\mathrm{x}} \in \mathrm{C}^{\mathrm{M} \times 1}$ is the corresponding additive zero mean gaussian white noise, then the following formula is specific illustration for $\mathbf{A}_{\mathrm{x}}$ :

$$
\begin{aligned}
\mathbf{a}_{\mathrm{x}, \mathrm{i}}\left(u_{\mathrm{x}}\right) & =\exp \left[\mathrm{j} 2 \pi \frac{2 P_{\mathrm{x}}-(i-1) d_{\mathrm{x}}}{\lambda \cdot R_{0}} x_{u_{\mathrm{x}}}(t)\right], \\
\mathbf{A}_{u_{\mathrm{x}}} & =\left[\mathbf{a}_{\mathrm{x}, 1}\left(u_{\mathrm{x}}\right), \cdots, \mathbf{a}_{\mathrm{x}, \mathrm{M}}\left(u_{\mathrm{x}}\right)\right]^{\mathrm{T}}, \\
\mathbf{A}_{\mathrm{x}} & =\left[\mathbf{A}_{\mathrm{x}, 1}, \cdots, \mathbf{A}_{\mathrm{x}, \mathrm{U}_{\mathrm{x}}}\right] .
\end{aligned}
$$

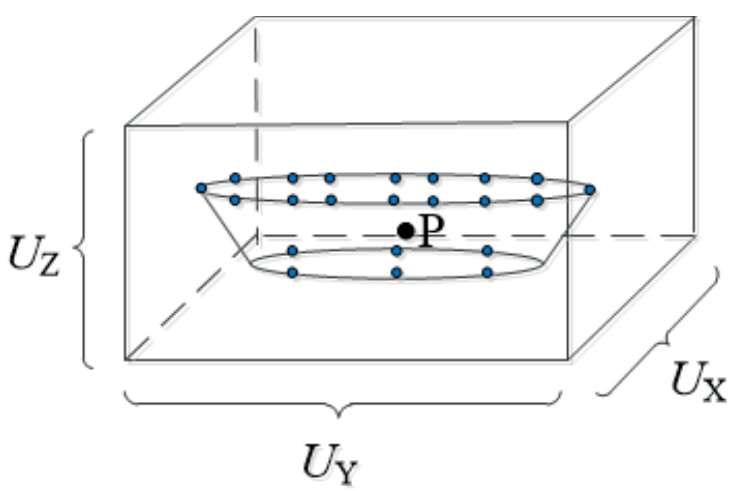

Fig. 4. The selected imaging space area. 
Then, due to the sparsity of $\boldsymbol{\alpha}_{\mathrm{x}}$ we choose maximum a posterior (MAP) method to estimate scattering vector $\boldsymbol{\alpha}_{\mathrm{x}}$, suppose $\mathbf{e}_{\mathrm{x}} \sim C N(0, \eta I)$, we can get the probability density of echo $\mathbf{D}_{\mathrm{X}}$ :

$$
P\left(\mathbf{D}_{\mathrm{x}} \mid \boldsymbol{\alpha}_{\mathrm{x}}, \eta\right)=(2 \pi \eta)^{-\mathrm{M}} \exp \left(-\left\|\mathbf{D}_{\mathrm{x}}-\mathbf{A}_{\mathrm{x}} \boldsymbol{\alpha}_{\mathrm{x}}\right\|_{2}^{2} / 2 \eta\right)
$$

Then, we can get priori probability based on the Laplace prior distribution of $\boldsymbol{\alpha}_{\mathrm{x}}$ :

$$
P\left(\boldsymbol{\alpha}_{\mathrm{x}} \mid \mu\right)=(\mu / 2) \cdot \exp \left(-\mu\left\|\boldsymbol{\alpha}_{\mathrm{x}}\right\|_{1} / 2\right)
$$

where $\mu$ is the coefficient of sparse distribution which is independent to $\alpha_{\mathrm{x}}$, then assume $P(\eta) \propto 1, P(\lambda) \propto 1$, according to $(21)$ and (22), the posterior probability density can be expressed as

$$
\begin{aligned}
& P\left(\boldsymbol{\alpha}_{\mathrm{x}}, \mu, \eta \mid \mathbf{D}_{\mathrm{x}}\right) \propto P\left(\boldsymbol{\alpha}_{\mathrm{x}}, \mu, \eta, \mathbf{D}_{\mathrm{x}}\right) \\
& =p\left(\mathbf{D}_{\mathrm{x}} \mid \boldsymbol{\alpha}_{\mathrm{x}}, \mu, \eta\right) p\left(\boldsymbol{\alpha}_{\mathrm{x}} \mid \mu\right) p(\eta) p(\mu) \\
& =(2 \pi \eta)^{-\mathrm{M}} \exp \left(-\left\|\mathbf{D}_{\mathrm{x}}-\mathbf{A}_{\mathrm{x}} \boldsymbol{\alpha}_{\mathrm{x}}\right\|_{2}^{2} / 2 \eta\right) \\
& \cdot(\mu / 2) \exp \left(-\mu\left\|\boldsymbol{\alpha}_{\mathrm{x}}\right\|_{1} / 2\right) .
\end{aligned}
$$

So we can get the cost function $\mathrm{f}$ by negative logarithm operation

$$
\begin{aligned}
& \max \left(-\ln \left(P\left(\boldsymbol{\alpha}_{\mathrm{x}}, \mu, \eta \mid \mathbf{D}_{\mathrm{x}}\right)\right)\right) \\
& =\min \left(M \ln (2 \pi \eta)+\frac{1}{2 \eta}\left\|\mathbf{D}_{\mathrm{x}}-\mathbf{A}_{\mathrm{x}} \boldsymbol{\alpha}_{\mathrm{x}}\right\|_{2}^{2}\right. \\
& \left.-\ln (\mu / 2)+\frac{\mu}{2}\left\|\boldsymbol{\alpha}_{\mathrm{x}}\right\|_{1}\right)=\min (f) .
\end{aligned}
$$

Thus the parameter estimation problem is transformed into a function extremum problem, we can get following result at $(s+1)$ iteration based on $\frac{\partial f}{\partial \alpha_{\mathbf{x}}{ }^{\mathrm{H}}}=0$ and $\frac{\partial f}{\partial \eta}=0$ :

$$
\begin{aligned}
& \boldsymbol{\alpha}_{\mathrm{x}}^{s+1}=\left(\mathbf{A}_{\mathrm{x}}{ }^{\mathbf{H}} \mathbf{A}_{\mathrm{x}}+\eta_{s} \boldsymbol{\Gamma}_{s}^{-1}\right)^{-1} \mathbf{A}_{\mathrm{x}}{ }^{\mathbf{H}} \mathbf{D}_{\mathrm{x}} \\
& \eta_{s+1}=\left\|\mathbf{D}_{\mathrm{x}}-\mathbf{A}_{\mathrm{x}} \boldsymbol{\alpha}_{\mathrm{x}}^{s}\right\|_{2}^{2} / M
\end{aligned}
$$

where

$$
\boldsymbol{\Gamma}=\left[\begin{array}{cccc}
\left|\alpha_{\mathrm{x}, 1}\right| & 0 & \cdots & 0 \\
0 & \left|\alpha_{\mathrm{x}, 2}\right| & \cdots & 0 \\
\vdots & \vdots & \ddots & \vdots \\
0 & 0 & \cdots & \left|\alpha_{\mathrm{x}, \mathrm{U}_{\mathrm{X}}}\right|
\end{array}\right]
$$

After same processing above, we can get approximate distribution of targets in the three dimensions, which can reduce the search range and realize sparse recovery. It should be noted that when realizing sparse imaging, the measurement should be firstly constructed according to (18). Unfortunately, the position of real target is difficult to fall on the pre-divided grid point, so the deviation between them will lead to off-grid problem. As for this, the paper adopts an approximate method, that is, selecting the point closest to the mismatched target point to replace the mismatched point, in order to reduce the imaging error.

\section{Simulation Results and Discussion}

The simulations aim to prove that MIMO radar can still achieve high resolution and accurate imaging even if the ship has pitch, roll and yaw rotations. The parameters of two radar systems are set as Tab. 1 and Tab. 2, the range of imaging area is also presented in Tab. 1. Moreover, in order to show the effects caused by target rotations more intuitively, the values of motion parameters are set as Tab. 3 .

In the simulation experiments, strong scattering points are set on the hull of the ship as shown in Fig. 4, and all of their scattering coefficients are set to 1 . Three dimensions are all divided into 100 grids. Then simulation results Figs. 5-8. can be obtained as following.

Figure 5 shows the 3-D MIMO radar imaging results of ship target with complex rotations when SNR is $10 \mathrm{~dB}$, where (a) is the initial position of ship target, (b)-(d) are 3-D imaging results when ship moves for $2 \mathrm{~s}, 3 \mathrm{~s}, 4 \mathrm{~s}$. In each figure, it is obvious that the 3-D motion states of ship target can be intuitively presented and the position of each target point can be accurately obtained. More importantly, slight changes of target motion or position can be captured in real time, which is significant for target recognition and motion prediction in practice.

\begin{tabular}{|c|c|}
\hline Parameters & Value \\
\hline Transmitting elements number & 10 \\
\hline Receiving elements number & $10 \times 10$ \\
\hline Transmitting array space & $8 \mathrm{~m}$ \\
\hline Receiving array space & $8 \mathrm{~m}$ \\
\hline Carrier frequency & $10 \mathrm{GHz}$ \\
\hline Waveform bandwidth & $50 \mathrm{MHz}$ \\
\hline Pulse width & $2 \mu \mathrm{s}$ \\
\hline Wavelength & $0.03 \mathrm{~m}$ \\
\hline Imaging range of X dimension & $2800 \mathrm{~m}-3000 \mathrm{~m}$ \\
\hline Imaging range of Y dimension & $4000 \mathrm{~m}-4200 \mathrm{~m}$ \\
\hline Imaging range of $Z$ dimension & $4900 \mathrm{~m}-5100 \mathrm{~m}$ \\
\hline
\end{tabular}

Tab. 1. Parameters of MIMO radar imaging.

\begin{tabular}{|c|c|}
\hline Parameters & Value \\
\hline Carrier frequency & $10 \mathrm{GHz}$ \\
\hline Waveform bandwidth & $50 \mathrm{MHz}$ \\
\hline Pulse width & $2 \mu \mathrm{s}$ \\
\hline Wavelength & $0.03 \mathrm{~m}$ \\
\hline Resolution & $2 \mathrm{~m} \times 5 \mathrm{~m}$ \\
\hline Velocity of radar carrier & $100 \mathrm{~m} / \mathrm{s}$ \\
\hline Synthetic aperture time & $7.3 \mathrm{~s}$ \\
\hline Data sampling length & $1024 \times 512$ \\
\hline
\end{tabular}

Tab. 2. Parameters of SAR imaging.

\begin{tabular}{|c|c|c|c|}
\hline Rotation & Amplitude[deg] & Period[sec] & Phase[rad] \\
\hline Pitch & 15 & 6 & -0.1 \\
\hline Roll & 20 & 10 & 0.1 \\
\hline Yaw & 10 & 5 & 0 \\
\hline
\end{tabular}

Tab. 3. Parameters of pitch, roll, yaw rotations. 


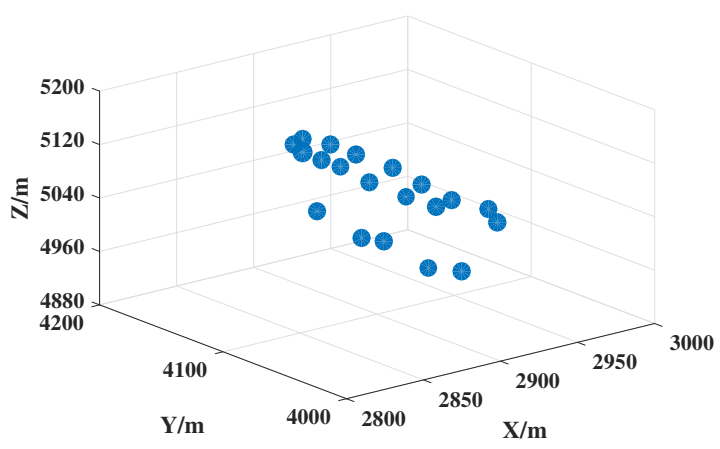

(a)

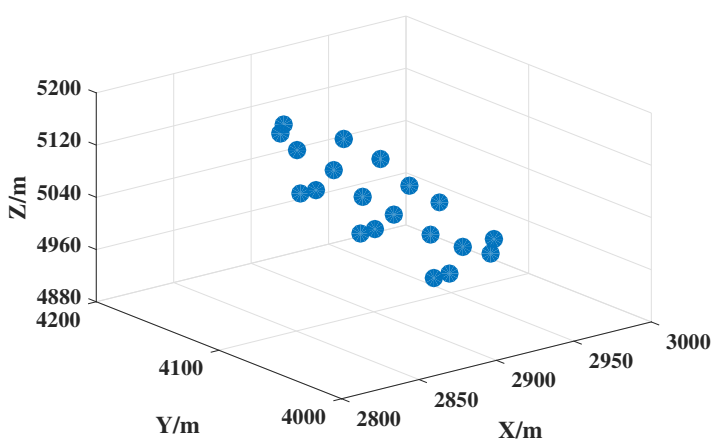

(c)

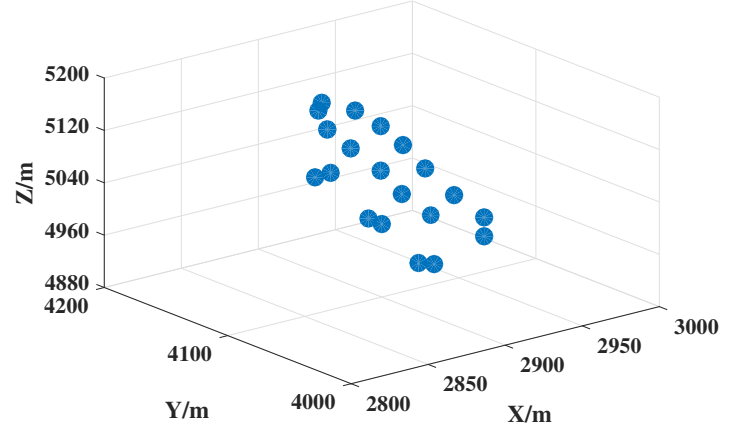

(b)

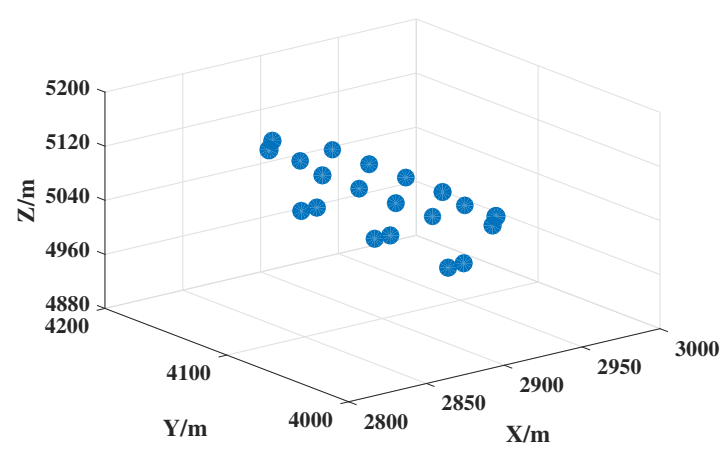

(d)

Fig. 5. 3-D MIMO radar imaging results. (a)-(d) are 3-D imaging results with ship moves $0 \mathrm{~s}, 2 \mathrm{~s}, 3 \mathrm{~s}, 4 \mathrm{~s}$.

Figure 6 shows the XY two-dimensional imaging for SAR with SNR $=10 \mathrm{~dB}$. (a) is the actual position and attitude of ship target, (b) is the imaging result applied the phase compensation method according to the literature [13]. The comparison shows that the compensation method is effective, and the real-time imaging can be realized. However, the accuracy of SAR imaging after compensation is obviously unable to meet the requirements, the imaging blurred problem can not be solved with high accuracy, the shape of the hull is still distorted with the changes of scattering coefficients. So it is difficult to estimate the accurate positions and motion states of real targets when SAR is used for ship target imaging with rotational motions.

Figure 7 shows the simulation results of MIMO radar imaging with yaw rotation considered when SNR is $10 \mathrm{~dB}$, where the true positions of targets are presented and the parameters of yaw rotation follow the settings in Tab. 3 Among them, (a) is the initial attitude of the ship, (b)-(f) are the imaging results after the ship rotates for $1 \mathrm{~s}, 2 \mathrm{~s}, 3 \mathrm{~s}, 4 \mathrm{~s}$ and $5 \mathrm{~s}$. Since the period of yaw rotation is 5 seconds, the ship will theoretically return to its initial state after 5 seconds without considering cruise motion, which can be verified from the results (a) and (f). Compared with Fig. 6, the strong realtime performance of MIMO radar imaging can be illustrated, which ensure the high accuracy. It should be noted that the estimation deviation is small and it is mainly caused by the approximate processing of the off-grid problem. The results show that MIMO radar is more suitable for more accurate ship target imaging with complex motion.

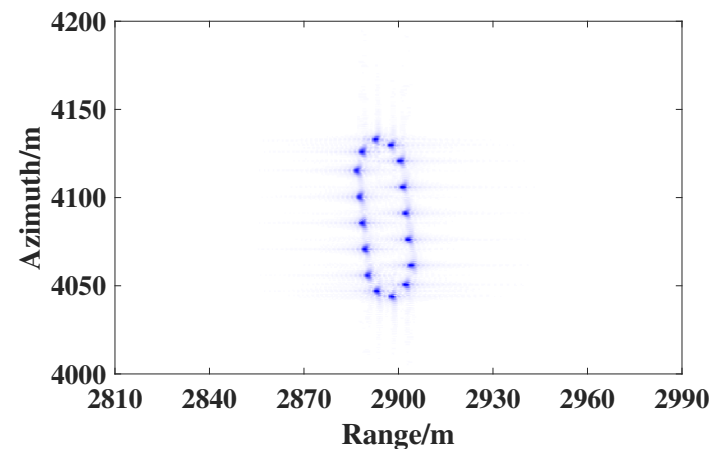

(a)

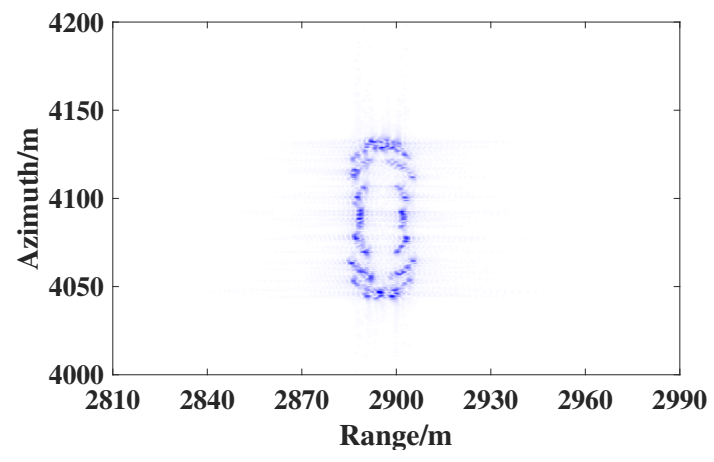

(b)

Fig. 6. SAR imaging results. (a) is the actual position of ship. (b) is SAR imaging result with phase correction and motion compensation. 


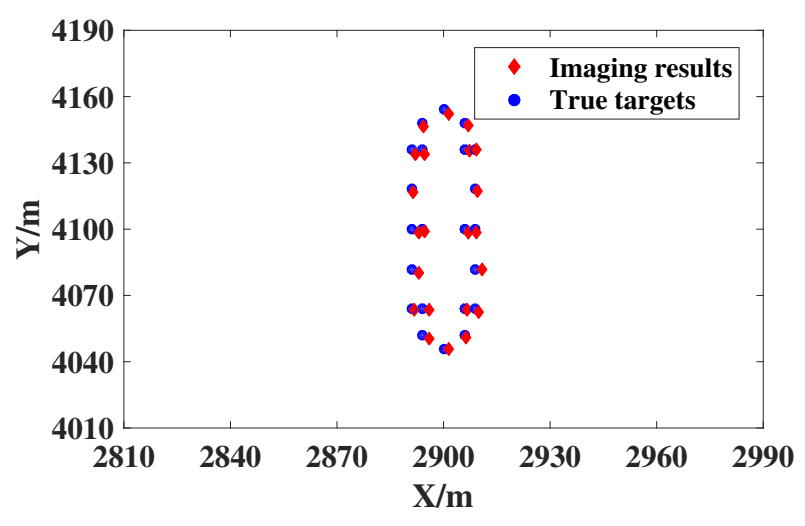

(a)

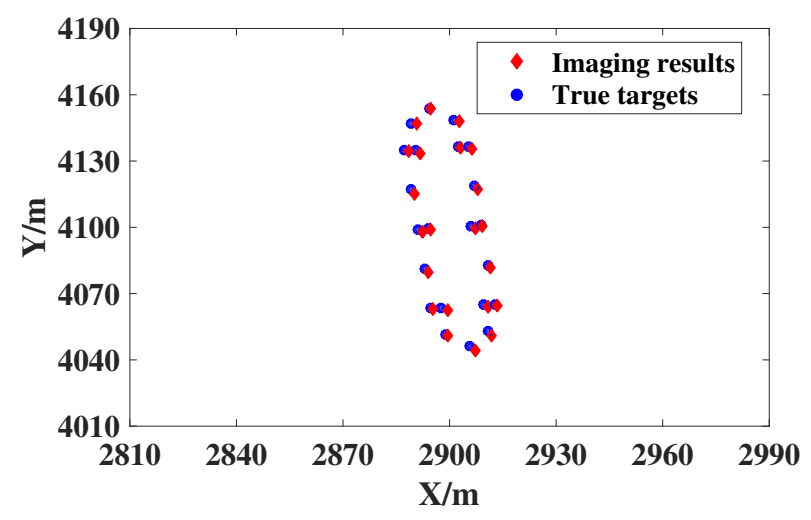

(c)

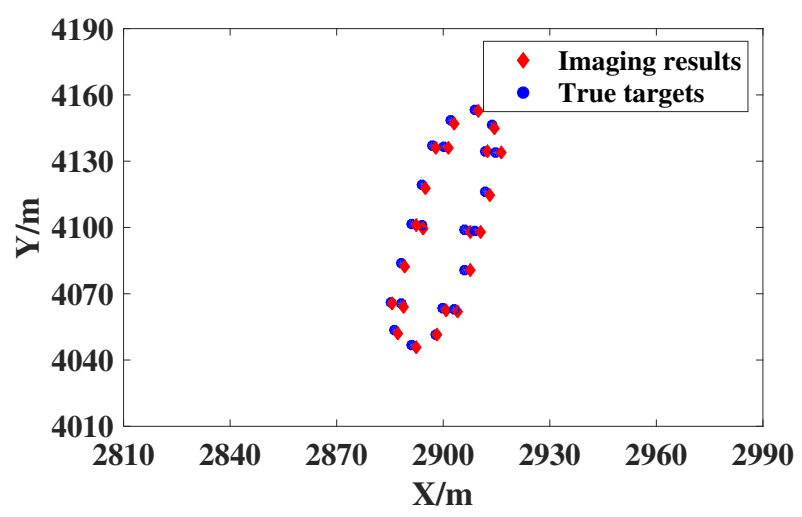

(e)

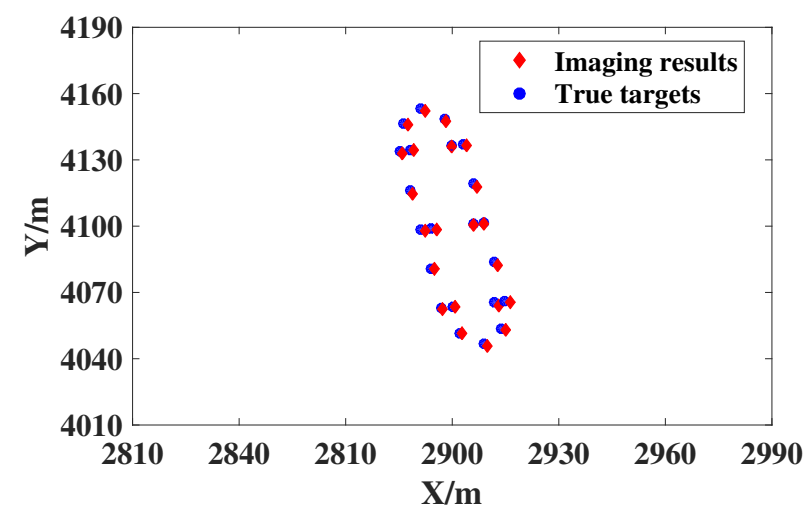

(b)

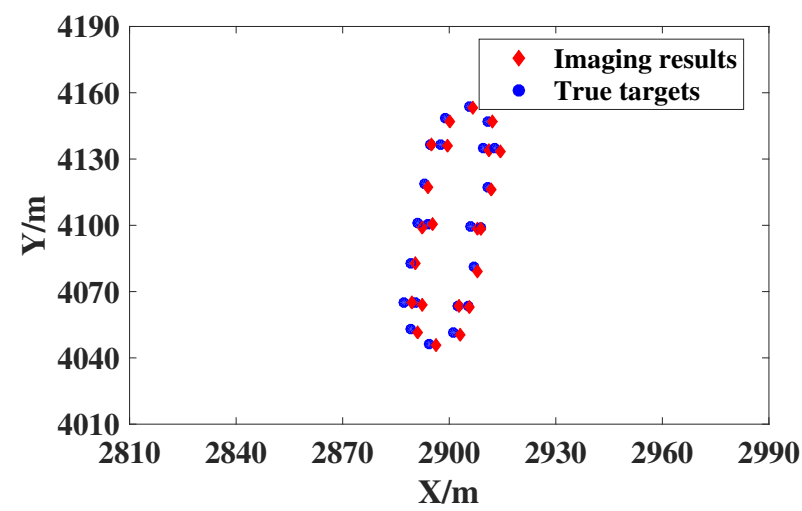

(d)

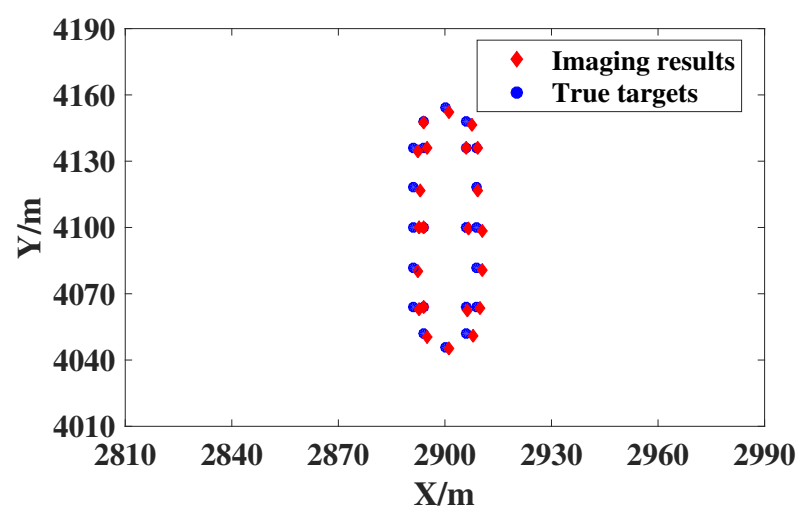

(f)

Fig. 7. MIMO radar imaging results for ship yaw rotation in a period. (a) is the initial position of ship. (b)-(f) respectively represent the imaging results with ship moves $1 \mathrm{~s}, 2 \mathrm{~s}, 3 \mathrm{~s}, 4 \mathrm{~s}, 5 \mathrm{~s}$

Figure 8 shows the accuracy performance of the proposed method. In simulation (a), SAR imaging method and the phase compensation method [13] are introduced for comparison with different SNR. It is obvious that the imaging is superior to other methods. (b) is the comparison result of imaging accuracy with Orthogonal Matching Puisuit method (OMP) and Dimension-reduction Kronecker CS method (DR-KCS) [21], which are commenly used in MIMO radar imaging. In the figure, accuracy performance of the proposed method is better than other methods, which benefits from the selection of appropriate prior information and the efficient estimation of noise. (c) describes the relationship between imaging accuracy and ship rotation amplitude. The horizontal axis in the figure represents the amplitude of the ship rotation, which actually reflects the changes of imaging accuracy in different application environments. The imaging error increases when the motion amplitude increases due to the deterioration of the environment. As shown in the figure, the imaging accuracy of MIMO radar is less affected by the environment when compared to other methods, which also proves that MIMO radar has high accuracy and stability. 


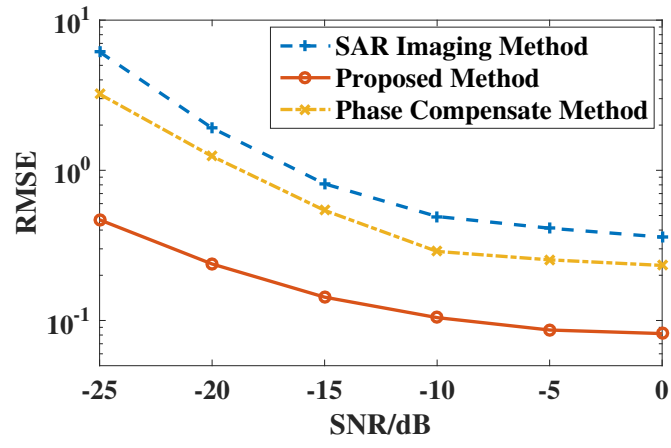

(a)

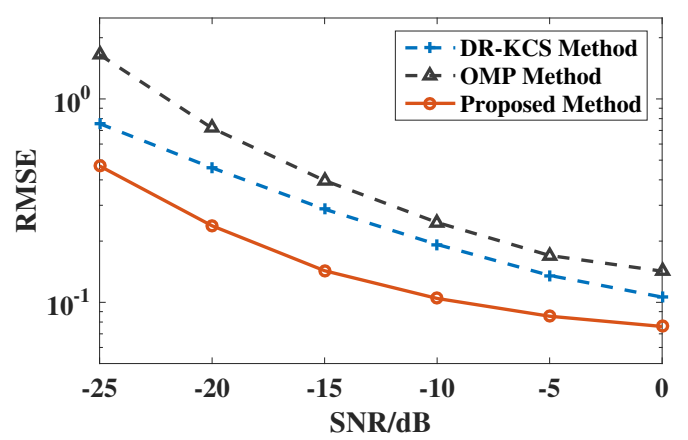

(b)

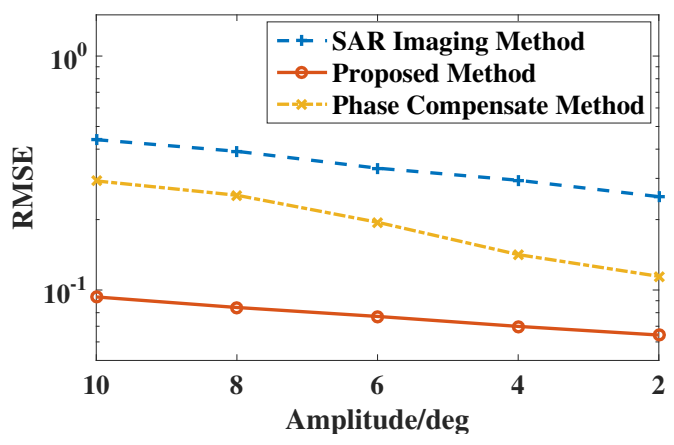

(c)

Fig. 8. Comparison of imaging accuracy in different conditions. (a) is accuracy comparison with SAR imaging and phase compensate method under different SNR. (b) is accuracy comparison with OMP method and DR-KCS method under different SNR. (c) is accuracy comparison with different yaw rotation amplitude.

\section{Conclusions}

In order to solve the problem of image defocusing or distortion in synthetic aperture radar (SAR) for ship target imaging with complex motion, a three-dimensional imaging method based on MIMO radar is proposed.In order to ensure the synchronization of the echo model and the ship attitude, this paper makes a detailed analysis of the image distortion in ship rotating motion, establishes a three-dimensional echo model, and obtains the scattering coefficient through geometric analysis, which ensures the real-time performance. Finally, MAP method is used to realize accurate imaging. Simulation results show that MIMO radar is more suitable for the imaging of ships with complex rotational motions.

\section{Acknowledgments}

This work is supported by the National Natural Science Foundation (61571148,61871143), Fundamental Research for the Central University (HEUCFG201823, 3072019CF0402), Heilongjiang Natural Science Foundation (LH2019F006), Research and Development Project of Application Technology in Harbin (2017R-AQXJ095).

\section{References}

[1] WARD, K. D. Radar imaging of ships at sea. In Proceedings of the IEE Colloquium on Synthetic Aperture Radar. London (UK), 1989, p. $7 / 1-7 / 5$.

[2] FINGAS, M. F., BROWN, C. E. Review of ship detection from airborne platforms. Canadian Journal of Remote Sensing, 2001, vol. 27 , no. 4, p. 379-385. DOI: 10.1080/07038992.2001.10854880

[3] REED, A. M., MILGRAM, J. H. Ship wakes and their radar images. Annual Review of Fluid Mechanics, 2002, vol. 34, p. 469-502. DOI: 10.1146/annurev.fluid.34.090101.190252

[4] CRISP, D. J. The state-of-the-art in ship detection in Synthetic Aperture Radar imagery. Defence Science and Technology Organisation Salisbury (Australia) Info Sciences Lab, No. DSTO-RR-0272, 2004, p. 3-22.

[5] CHEN, C.-C., ANDREWS, H. C. Target-motion-induced radar imaging. IEEE Transactions on Aerospace and Electronic Systems, 1980, vol. AES-16, no. 1, p. 2-14. DOI: 10.1109/TAES.1980.308873

[6] OUCHI, K., IEHARA, M., MORIMURA, K., et al. Nonuniform azimuth image shift observed in the Radarsat images of ships in motion. IEEE Transactions on Geoscience and Remote Sensing, 2002, vol. 40, no. 10, p. 2188-2195. DOI: 10.1109/TGRS.2002.802478

[7] MATGERIT, G., MALLORQUI, J. J., RIUS, J. M., et al. On the usage of GRECOSAR, an orbital polarimetric SAR simulator of complex targets, to vessel classification studies. IEEE Transactions on Geoscience and Remote Sensing, 2006, vol. 44, no. 12, p. 3517-3526. DOI: $10.1109 / \operatorname{tgrs} .2006 .881120$

[8] COCHIN, C., LOUVIGNE, J. C., FABBRI, R., et al. Radar simulation of ship at sea using MOCEM V4 and comparison to acquisitions. In Proceedings of the International Radar Conference. Lille (France), 2014, p. 1-6. DOI: 10.1109/RADAR.2014.7060241

[9] LAZAROV, A. D., MINCHEV, C. N. SAR ship target imaging by induced complementary movement. In Proceedings of the 4th International Conference on Recent Advances in Space Technologies. Istanbul (Turkey), 2009, p. 441-446. DOI: 10.1109/RAST.2009.5158242

[10] CAI, Y., CHONG, J. The study of SAR-ISAR ship imaging based on time-frequency analysis. In Proceedings of the IEEE Youth Conference on Information, Computing and Telecommunication. Beijing (China), 2009, p. 158-161. DOI: 10.1109/YCICT.2009.5382402

[11] LIU, P., JIN, Y. Q. Simulation of synthetic aperture radar imaging of dynamic wakes of submerged body. IET Radar, Sonar and Navigation, 2016, vol. 11, no. 3, p. 481-489. DOI: 10.1049/iet-rsn.2016.0297

[12] BACKSTORM, V., SKARBRATT, A. Maritime ISAR Imaging with Airborne Radar. Master of Science Thesis, Chalmmers University of Technology, Goteborg (Sweden), 2010. [Online] Available at: http://publications.lib.chalmers.se/records/fulltext/129953.pdf 
[13] LIU, P., JIN, Y. Q. A study of ship rotation effects on SAR image. IEEE Transactions on Geoscience and Remote Sensing, 2017, vol. 55, no. 6, p. 3132-3144. DOI: 10.1109/TGRS.2017.2662038

[14] FISHLER, E., HAIMOVICH, A. M., BLUM, R. S. MIMO radar: An idea whose time has come. In Proceedings of the IEET International Radar Conference. Philadelphia (USA), 2004, p. 71-78. DOI: 10.1109/NRC.2004.1316398

[15] HAIMOVICH, A. M., BLUM, R. S., CIMINI, L. J. MIMO radar with widely separated antennas. Signal Processing Magazine, 2008, vol. 25, no. 1, p. 116-129. DOI: 10.1109/MSP.2008.4408448

[16] SHARMA, G. V. K., RAJA RAJESWARI, K. Fast implementation of transmit beamforming for colocated MIMO radar. Radioengineering, 2015, vol. 24, no. 1, p. 171-177. DOI: 10.13164/re.2015.0171

[17] HUANG, P., LI, X., WANG, H. Tensor-based match pursuit algorithm for MIMO radar imaging. Radioengineering, 2018, vol. 27, no. 2, p. 580-586. DOI: $10.13164 /$ re.2018.0580

[18] ZHU, Y. T., SU, Y. A type of M2-transmitter N2-recevier MIMO radar array and 3D imaging theory. Science China, 2011, vol. 54, no. 10 , p. 2147-2157. DOI: 10.1007/s11432-011-4400-y

[19] MA, C. Z., YEO, T., TAN, C. Three-dimensional imaging of targets using collocated MIMO radar. IEEE Transactions on Geoscience and Remote Sensing, 2011, vol. 49, no. 8, p. 3009-3021. DOI: 10.1109/TGRS.2011.2119321

[20] DING, S. S., TONG, N. N., ZHANG, Y. S., et al. Super-resolution 3D imaging in MIMO radar using spectrum estimation theory. IET Radar Sonar and Navigation, 2017, vol. 11, no. 2, p. 304-312. DOI: 10.1049/iet-rsn.2016.0233

[21] HU, X. W., TONG, N. N., ZHANG, Y. S., et al. Multipleinput-multiple-output radar superresolution three-dimensional imaging based on a dimension-reduction compressive sensing. IET Radar Sonar and Navigation, 2016, vol. 10, no. 4, p. 757-764. DOI: 10.1049 /iet-rsn.2015.0345

[22] HU, X. W., TONG, N. N., GUO, Y. D., et al. MIMO radar 3D imaging based on multi-dimensional sparse recovery and signal support prior information. IEEE Sensors Journal, 2018, vol. 18, no. 8, p. 3152-3162. DOI: 10.1109/JSEN.2018.2810705
[23] HAN, L., TIAN, B., FENG, C., et al. Extraction micro-Doppler feature of precession targets in orthogonal frequence division multiplexing-linear frequence modulation multiple input multiple output radar. In Proceedings of the International Conference on Systems and Informatics. Nanjing (China), 2018, p. 883-888. DOI: 10.1109/ICSAI.2018.8599377

[24] PASTINA, D., SANTI, F., BUCCIARELLI, M. MIMO distributed imaging of rotating targets for improved 2-D resolution. IEEE Geoscience and Remote Sensing Letters, 2015, vol. 12, no. 1, p. 190-194. DOI: 10.1109/LGRS.2014.2331754

[25] MARTINEZ, J., THURN, K., VOSSIEK, M. MIMO radar for supporting automated rendezvous maneuvers with non-cooperative satellites. In Proceedings of the IEEE Radar Conference. Seattle (USA), 2017, p. 497-501. DOI: 10.1109/RADAR.2017.7944254

\section{About the Authors ...}

Wei WANG was born in 1979, received his Ph.D. degree from Harbin Engineering University (HEU), Harbin, China in 2006. He is currently a professor in the Automation College in HEU. His research interests include MIMO radar signal processing, integrated navigation system and radio navigation technology.

Ziying HU was born in 1994, received his B.Sc. degree in 2016 from Harbin Engineering University, where he is currently pursuing the Ph.D. degree. His research interests include MIMO radar signal processing and the sparse imaging technology.

Ping HUANG (corresponding author) received his Ph.D. degree from Harbin Engineering University (HEU), Harbin, China in 2010. He is currently a associate professor in the Automation College in HEU. His research interests include ship navigation and sensing technology, satellite communication and radio navigation. 